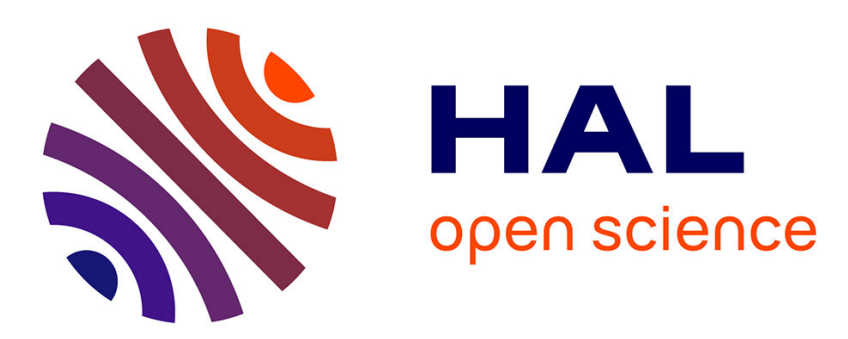

\title{
Towards disease-specific speech markers for differential diagnosis in Parkinsonism
}

\author{
Biswajit Das, Khalid Daoudi, Jiri Klempir, Jan Rusz
}

\section{To cite this version:}

Biswajit Das, Khalid Daoudi, Jiri Klempir, Jan Rusz. Towards disease-specific speech markers for differential diagnosis in Parkinsonism. ICASSP 2019 - IEEE International Conference on Acoustics, Speech, and Signal Processing, May 2019, Brighton, United Kingdom. hal-02103829

\section{HAL Id: hal-02103829 \\ https://hal.inria.fr/hal-02103829}

Submitted on 18 Apr 2019

HAL is a multi-disciplinary open access archive for the deposit and dissemination of scientific research documents, whether they are published or not. The documents may come from teaching and research institutions in France or abroad, or from public or private research centers.
L'archive ouverte pluridisciplinaire HAL, est destinée au dépôt et à la diffusion de documents scientifiques de niveau recherche, publiés ou non, émanant des établissements d'enseignement et de recherche français ou étrangers, des laboratoires publics ou privés. 


\title{
TOWARDS DISEASE-SPECIFIC SPEECH MARKERS FOR DIFFERENTIAL DIAGNOSIS IN PARKINSONISM
}

\author{
Biswajit Das ${ }^{1}$, Khalid Daoudi $^{1 *}$, Jiri Klempir $^{2}$, Jan Rusz $^{2,3 \dagger}$ \\ ${ }^{1}$ INRIA Bordeaux Sud-Ouest (GeoStat team) \\ Talence, France \\ ${ }^{2}$ Department of Neurology and Center of Clinical Neuroscience, First Faculty of Medicine \\ Charles University, Prague, Czech Republic \\ ${ }^{3}$ Department of Circuit Theory. Faculty of Electrical Engineering \\ Czech Technical University in Prague, Czech Republic \\ biswajit.das@inria.fr,khalid.daoudi@inria.fr,jiri.klempir@lf1.cuni.cz,rusz.mz@gmail.com
}

\begin{abstract}
Parkinsonism refers to Parkinson's Disease (PD) and Atypical Parkinsonian Syndromes (APS), such as Progressive Supranuclear Palsy (PSP) and Multiple System Atrophy (MSA). Discrimination between PD and APS and within APS groups in early disease stages is a very challenging task. Interestingly, speech disorder is frequently an early and prominent clinical feature of both PD and APS. This renders speech/voice analysis a promising tool for the development of an objective marker to assist neurologists in their diagnosis. This paper is a continuation of a recent work on speech-based differential diagnosis within APS. We address the difficult problem of defining disease-specific speech features which is crucial in the perspective of early differential diagnosis. We investigate this problem by considering the constraint that only a small amount of training data can be available in this setting. To do so, we perform univariate statistical analysis followed by a supervised learning that forces the designed new features to be 1-dimensional. We carry out experiments using speech recordings of MSA and PSP patients. We show that linear classification models allow the definition of new scalar variables which can be considered as speech features which are specific to each disease, MSA and PSP.
\end{abstract}

Index Terms - Pathological speech processing, Speech disorder, Parkinsonism, Parkinson's disease, Machine learning

\section{INTRODUCTION}

Speech requires the integrity and integration of numerous neurocognitive, neuromotor, neuromuscular and muscu-

\footnotetext{
${ }^{*}$ This work is partly funded by the French research agency (ANR), project Voice4PD-MSA n ${ }^{\circ}$ ANR-16-CE19-0010-01.

${ }^{\dagger}$ This work is partly funded by the Research Center for Informatics ( grant nr. CZ.02.1.01/0.0/0.0/16_019/0000765), and by the Czech Ministry of Education (PROGRES-Q27/LF1).
}

loskeletal activities [1]. Deficit in any of this activity may be the cause of speech disorder. It is an early and prominent indication for different neurological disorder. Therefore, in such disorders, recognizing and understanding predictable patterns of speech disturbances and their underlying neurophysiological bases can help to understand nervous system organization for speech motor control.

Parkinson's disease (PD) is a neurological disorder caused by the degeneration of dopaminergic neurons, leading to clinical features characterized by bradykinesia, rigidity, resting tremor and postural instability. Atypical parkinsonian syndromes (APS) such as progressive supranuclear palsy (PSP) and multiple system atrophy (MSA) differ from PD by more widespread neuronal involvement, resulting in additional clinical signs, more rapid disease progression and poor response to dopamine replacement therapy [2]. The majority of PSP and MSA patients develop clinical features that overlap those of PD and thus the correct diagnosis can be very challenging in early stages of the disease. However, an accurate early diagnosis is essential not only in assessing prognosis and making decisions regarding treatment, but also for understanding the underlying pathophysiology and for the development of new therapies [3].

It turns out that speech impairment is frequently an early and prominent clinical feature of PD as well as APS. During the last decades, there has been a increasing interest in PD speech and voice analysis [4]. However, very few attempts have been done in differential diagnosis between PD and APS or within APS [5, 6, 7, 8, 9]. A pioneer work [10] provides a quantitative and objective analysis of speech characteristics for the discrimination between PD and APS and between MSA and PSP. The basic conclusion is that PD speakers manifest pure hypokinetic dysarthria, ataxic components are more affected in MSA whilst PSP subjects demonstrate severe deficits in hypokinetic and spastic elements of dysarthria. Using an SVM with a Gaussian radial basis kernel 
and an exhaustive search, [10] reported a 95\% accuracy in objective discrimination between APS and PD and 75\% in discrimination between PSP and MSA. We emphasize that classification performance was not the main purpose of [10], but rather a way to seek disease-specific dysarthric signs. In our recent study [11], standard linear and generalized linear models were explored to address the curse of dimensionality problem in this setting. This study led to an $80 \%$ accuracy in classification between MSA and PSP.

This paper is a continuation of our previous work on discrimination between PSP and MSA [11]. Here, we focus on defining new speech features which can objectively measure particular dysarthria attributes and which are disease-specific, in the sense that such features would have a (statistical) behavior for PSP which is significantly different than for MSA. Moreover, we want such features to be clinically interpretable in order to improve the understanding of speech impairments in PSP and MSA. Obviously, the first benefit of such investigation would be accurate and objective discrimination between PSP and MSA, given that subjective evaluation is quite challenging due to similar perceptual behavior [12]. The second and more important benefit is to potentially allow drawing hypothesis regarding the early stage of the diseases.

The paper is organized as follows. Section 2 describes the speech database. Acoustic features used in this study are described in Section 3. Methodology and experiments are presented in Section 4.

\section{DATASET}

From 2011 to 2014,12 consecutive patients with the clinical diagnosis of probable PSP (10 men, 2 women) and 13 patients with the diagnosis of probable MSA ( 6 men, 7 women) were recruited. In this series, 9 PSP patients were diagnosed with the Richardson's syndrome (PSP-RS), 2 with PSPparkinsonism (PSP-P) and 1 with PSP-pure akinesia with gait freezing (PAGF), whereas $10 \mathrm{MSA}$ patients were diagnosed as the parkinsonian type (MSA-P) and 3 as cerebellar type (MSA-C). The diagnosis of PSP was established by the NINDS-PSP clinical diagnosis criteria [13], MSA according to consensus diagnostic criteria for MSA [14]. Speech severity did not perceptually differ between PSP and MSA based on UPDRS speech item 18. For a detailed description of the patients we refer to [10].

Speech recordings were performed in a quiet room with a low ambient noise level using a head-mounted condenser microphone (Bayerdynamic Opus 55, Heilbronn, Germany) situated approximately $5 \mathrm{~cm}$ from the mouth of each subject. Speech signals were recored with $48 \mathrm{kHz}$ sampling frequency and 16-bit resolution. Each participant was instructed to perform sustained phonation of the vowel /a/ per one breath as long and steadily as possible, fast /pa/-/ta/-/ka/ syllable repetition at least seven times per one breath, a reading passage and a monologue on a given topic for approximately 90 s. All

\begin{tabular}{|c|c|c|}
\hline Speech features & Vocal task & Description \\
\hline \multicolumn{3}{|l|}{ Hypokinetic: } \\
\hline 1. Harsh voice & $\begin{array}{l}\text { Sustained phonation } \\
/ \mathrm{a} /\end{array}$ & $\begin{array}{l}\text { Jitter:Frequency perturbation; Shim- } \\
\text { mer:Amplitude perturbation; Harmonics- } \\
\text { to-noise ratio (HNR): amount of noise in } \\
\text { voiced speech }\end{array}$ \\
\hline $\begin{array}{l}\text { 2. Rapid Alternating } \\
\text { Motion Rate (AMR) }\end{array}$ & Syllable repetition & $\begin{array}{l}\text { It is measured as pace acceleration; It } \\
\text { provides impression of rapid and blurred } \\
\text { speech }\end{array}$ \\
\hline \multicolumn{3}{|l|}{$\begin{array}{l}\text { 3. Inappropriate si- } \\
\text { lences }\end{array}$} \\
\hline $\begin{array}{l}\text { i) Percent pause } \\
\text { time (PPT) }\end{array}$ & Reading passages & $\begin{array}{l}\text { PPT is measured as the percentage of pause } \\
\text { time relative to total speech time }\end{array}$ \\
\hline \begin{tabular}{lll}
\multicolumn{1}{c}{ ii) } & Number & of \\
pauses & (No. & of \\
pauses) & &
\end{tabular} & Reading passages & $\begin{array}{l}\text { No. of pauses measured as the average } \\
\text { number of pauses per second }\end{array}$ \\
\hline $\begin{array}{l}\text { iii) Intra-word } \\
\text { pause ratio }\end{array}$ & Reading passages & $\begin{array}{l}\text { The intra-word pause ratio measured as the } \\
\text { ratio between the total pause time within } \\
\text { polysyllabic words and the total pause } \\
\text { time. }\end{array}$ \\
\hline 5. Monopitch & Monologue & $\begin{array}{l}\text { Monotone voice, lacking normal pitch and } \\
\text { inflection changes }\end{array}$ \\
\hline \multicolumn{3}{|l|}{ Spastic: } \\
\hline $\begin{array}{l}\text { 1. The degree of } \\
\text { voicelessness (DUV) }\end{array}$ & $\begin{array}{l}\text { Sustained phonation } \\
/ \mathrm{a} /\end{array}$ & $\begin{array}{l}\text { DUV represents the fraction of pitch } \\
\text { frames marked as unvoiced. }\end{array}$ \\
\hline 2. Slow AMR & Syllable repetition & $\begin{array}{l}\text { It is measured as the DDK rate of the first } \\
\text { seven repetitions of the } / \mathrm{pa} / / \mathrm{ta} /-/ \mathrm{ka} / \text { sylla- } \\
\text { bles. }\end{array}$ \\
\hline \multicolumn{3}{|l|}{ Ataxic: } \\
\hline $\begin{array}{l}\text { 1. The excess pitch } \\
\text { fluctuation (F0_SD) }\end{array}$ & $\begin{array}{l}\text { Sustained phonation } \\
/ \mathrm{a} /\end{array}$ & $\begin{array}{l}\text { Pitch fluctuation measured as the standard } \\
\text { deviation of voice pitch }\end{array}$ \\
\hline 2. Irregular AMR & Syllable repetition & $\begin{array}{l}\text { It is measured as the standard deviation of } \\
\text { distances between consecutive positions of } \\
\text { syllables in the first seven repetitions of } \\
/ \mathrm{pa} /-/ \mathrm{ta} / / \mathrm{ka} /\end{array}$ \\
\hline 3. Vocal tremor & $\begin{array}{l}\text { Sustained phonation } \\
\text { /a/ }\end{array}$ & $\begin{array}{l}\text { Vocal tremor is measured as the frequency } \\
\text { tremor intensity index (FTRI) defined as } \\
\text { the intensity/magnitude of the strongest } \\
\text { low-frequency modulation of F0 [19]. }\end{array}$ \\
\hline
\end{tabular}

Table 1. List and description of the 13 features, grouped by dysarthria type

participants performed the sustained phonation and syllable repetition tasks twice.

\section{ACOUSTIC FEATURES}

In earlier studies $[15,16]$ several acoustic parameters are explored, we refer to [17] for the detailed description. In the same manner as in our recent study [11], in order to allow easy future comparisons or reproduction, we consider in the paper the same set of 13 features that can be computed with existing and established scripts. In [11], we adopted a "phonetic" point of view to group the feature because our main concern was the investigation the usability of linear models to address the curse of dimensionality. In this work, our concern is to find/define features which can be specific to each disease and which can lead to a clinical interpretation. We thus adopt a "symptomatic" point of view (as in [10]) to categorize the features into the well-known 3 dysarthria groups: Hypokinetic, Spastic and Ataxic. The set of the 13 features we consider is presented in Table 1. All there features are computed using Python and Praat scripts [18]. For phonation and syllable repetition, the final value of each feature is calculated as the mean of the values obtained from two vocal tasks. 
In hypokinetic dysarthria, abnormality is most notably observed in respiratory or aerodynamic function, laryngeal function, and motor control. It leads to reduced vocal loudness, monopitch, disruptions of voice quality and abnormal fast rate of speech [1]. Conversely, in ataxic dysarthria, loudness may vary excessively, and increased effort is evident which can be described as explosive speech, pitch variation, irregular AMR and vocal tremor are notable components of this group. On the other hand, spastic dysarthria can be demonstrated as voice with strained or strangled, pitch breaks. Therefore, degree of voicelessness and slow AMR are grouped in spastic dysarthria.

\section{METHODOLOGY AND EXPERIMENTS}

In all experiments, the MSA and PSP data is used to normalize the acoustic features to zero mean and unit variance.

\subsection{Univariate statistical analysis}

Given that we are faced with the curse of dimensionality problem, we need to perform feature selection and/or reduction. The simplest way to do feature selection is to carry out a univariate statistical analysis. We use the latter as a first pass to discard the highly correlated (thus less discriminative) features. To do so, for each feature, we first compute the intra MSA (resp. PSP) class mean $\mu_{m s a}$ (resp. $\mu_{p s p}$ ) and variance $\sigma_{m s a}$ (resp. $\sigma_{p s p}$ ). We then use Mahalanobis distance, defined in Equation 1, as a measure of the the inter-class separation.

$$
D=\sqrt{\left(\mu_{p s p}-\mu_{m s a}\right)^{2} \cdot\left(\frac{\sigma_{p s p}+\sigma_{m s a}}{2}\right)^{-1}}
$$

The result of this univariate analysis is given in Figure 1. This analysis suggests that we can fairly discard monopitch from the hypokinetic set of features. Likewise, this analysis suggests that DUV and slow AMR can be disregarded. We will keep them however in our second stage of analysis in order to check whether this "first indication" stands in our second stage of analysis. As for the ataxic group, it seems like all features are contributing towards discrimination. We thus end up considering the following sets of features:

- $\mathrm{H}=\{$ jitter, shimmer, HNR, intra-word pause, rapid AMR, no. of pauses, PPT $\}$

- $\mathrm{A}=\left\{\mathrm{F} 0 \_\mathrm{SD}\right.$, irregular AMR, vocal tremor $\}$

- $\mathrm{S}=\{\mathrm{DUV}$, slow AMR $\}$

\subsection{Learning a new speech feature}

In this section, we perform our second stage of feature selection and dimension reduction. Nowadays, most of "hot" machine learning problems and methods deal with large data

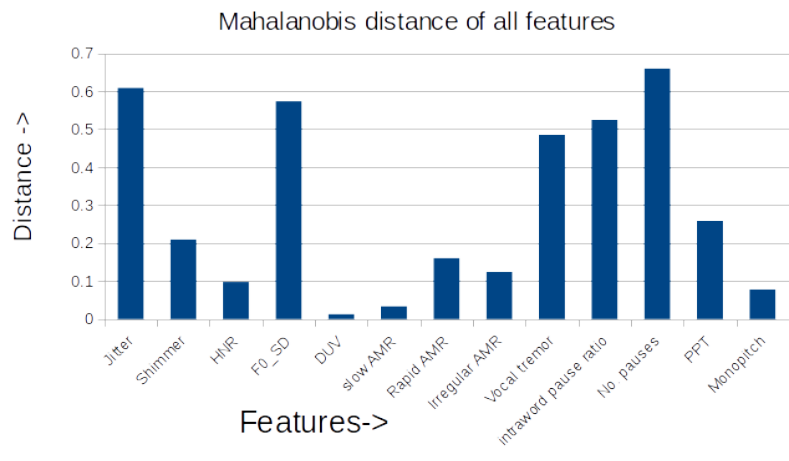

Fig. 1. Feature-wise distance between PSP and MSA

\begin{tabular}{||l|c|c|c||}
\hline & H & A & S \\
\hline \hline Accuracy (\%) & 68 & 8 & 0 \\
\hline
\end{tabular}

Table 2. Classification accuracy for individual dysarthric groups

sets (big data problems). In many applications however, such as biomedical engineering, data are rare and/or are collected with a (very) low time resolution. The problem we address in this paper falls in this setting; we are in a small dataset machine learning scenario. Indeed, only 12 PSP and 13 MSA patients are available. Thus, typically only a 1-dimensional feature space may provide acceptable statistics. Univariate statistical analysis, as performed in the previous section, is one solution. In fact, a more involved univariate analysis has been carried out [10], using the same dataset. This led to the argument that ataxic components are more affected in MSA whilst PSP subjects demonstrate severe deficits in hypokinetic and spastic elements of dysarthria. However, univariate analysis does not allow us to achieve the goal we target in this paper, that is finding a scalar speech feature which is disease specific and which is clinically interpretable.

In [11], we presented a methodology to deal with the machine learning part of our small dataset problem. We showed that classical linear and generalized linear models, such as Factorial Discriminant Analysis (FDA) (also known as descriptive LDA) [20], can provide a simple solution to this problem. We thus proceed now to investigate whether such models can lead us to our goal.

For classification, as the amount of data is small, a LeaveOne-Speaker-Out (LOSO) training approach is adopted in all the experiments. In order to have a clear understanding of

\begin{tabular}{||c|c|c|c|c||}
\hline & $\mathrm{H}+\mathrm{S}+\mathrm{A}$ & $\mathrm{H}+\mathrm{S}$ & $\mathrm{S}+\mathrm{A}$ & $\mathrm{H}+\mathrm{A}$ \\
\hline \hline Accuracy (\%) & 72 & 60 & 24 & $\mathbf{8 4}$ \\
\hline
\end{tabular}

Table 3. Classification accuracy for combined dysarthric groups 


\begin{tabular}{|c||c|c|c|c|c|c|c||c|c|c||}
\hline \multicolumn{1}{|c||}{} & \multicolumn{9}{c||}{ Hypokinetic } & \multicolumn{3}{c||}{ Ataxic } \\
\hline & Jitter & Shimmer & HNR & Intra-word pause & No. of pauses & PPT & Rapid AMR & F0_SD & Irregular AMR & Vocal tremor \\
\hline \hline Weight & 1.03 & 3.02 & 4.77 & -0.10 & -0.02 & -1.11 & 0.80 & 2.14 & -0.49 & 0.37 \\
\hline
\end{tabular}

Table 4. Feature weights obtained by FDA

the data behavior, we use a simple Gaussian classifier and a $1 d$ linear Support Vector Machine (SVM) with $C=1$ as classifiers. The results we present in the following are the same using these two classifiers.

We start (naturally) by evaluating FDA on each dysarthric group $(\mathrm{H}, \mathrm{A}, \mathrm{S})$ individually. The idea here is to check whether a linear combination of a group feature can discriminate between MSA and PSP. Table 2 shows the FDA classification scores between PSP and MSA patients by individual dysarthric group. This result shows that FDA on A and $\mathrm{S}$ are extremely low and even worse than chance. This suggest that (linear combination of) the ataxic and spastic features we consider cannot be individually disease-specific. The same argument holds for hypokinetic features, though the score is significantly higher.

We now proceed to evaluate the combination of dysarthric groups. Table 3 presents the FDA classification scores for the 4 possible combinations. This result shows that the same argument we made for individual group holds also for $\mathrm{H}+\mathrm{S}+\mathrm{A}$, $\mathrm{H}+\mathrm{S}$ and $\mathrm{S}+\mathrm{A}$. Interestingly the score obtained by $\mathrm{H}+\mathrm{A}$ is significantly high, even higher than the score in our recent work. This is a very good indication that measuring the "mutual amount" of hypokinetic and ataxic dysarthria can allow discrimination between MSA and PSP. Moreover, our result show that a relatively simple (weighted) averaging can measure this amount.

We now go deeper in the analysis of this averaging. Table 4 shows the weights obtained by FDA when training using all data (no LOSO). By looking at the weights, one observes that intra-word pause and no. of pauses have lower weights compared to the other feature. This suggests that they can be discarded in the linear FDA projection. We did so and rerun a LOSO training by discarding intra-word pause and no. of pause. This yields a classification score of $\mathbf{8 8 \%}$. This means that the arguments we stretched about hypokinetic and ataxic features $(\mathrm{H}+\mathrm{A})$ hold even better when discarding these two hypokinetic features. Figure 2 shows the values of the resulting variable for PSP (blue) and MSA (green) patients. The latter shows that a very good separation is indeed obtained. We mention however that these classification scores should be considered with precaution because of the LOSO bias (different weights at each iteration).

\section{CONCLUSION}

In this paper, we addressed the difficult problem of defining disease-specific speech features which is crucial in the perspective of early differential diagnosis in Parkinsonism. We

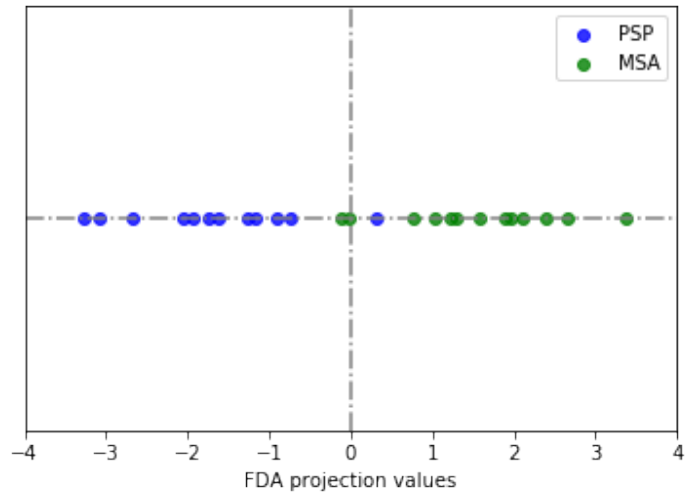

Fig. 2. Values of the new speech feature for each patient

focused on MSA and PSP and investigated this problem under the constraint of small dataset machine learning. Using FDA, we ended up defining a new scalar variable which can be considered as a disease-specific feature. This variable is learned from data as a linear combination of some hypokinetic and ataxic features. Using Gaussian or 1d SVM classifiers, we obtain a threshold which measures a certain degree of hypokinetic and ataxic "impairment". Above (resp. below) this threshold, the patient is classified as MSA (resp. PSP). Using LOSO training, we achieved $88 \%$ classification accuracy, which is an improvement w.r.t the $80 \%$ obtained in our recent work [11]. This achievement can be considered very successful as PSP and MSA are hardly perceptually distinguishable [12]. This result also suggests that hypokinetic and ataxic dysarthria convey considerable discriminative information when mutually considered. Moreover, it suggests that the hypokinetic and ataxic features we retained can be used as a vehicle to capture this information. We showed indeed that an appropriate weighted averaging of these features (the new variable) led to a high classification accuracy. We emphasize however that these arguments are not a "hard" conclusion. They need indeed to be confirmed by additional data and studies. This is the purpose of our ongoing research. We are actually in the process of collecting a (relatively) large speech corpus of French PD and APS patients (in the framework of a large pilot study involving French university hospitals).

\section{REFERENCES}

[1] J.R. Duffy, Motor Speech Disorders Substrates, Differential Diagnosis, and Management, Elsevier, 2013. 
[2] A. Schrag, Y. Ben-Shlomo, and N.P. Quinn, "Prevalence of progressive supranuclear palsy and multiple system atrophy: a cross-sectional study," The Lancet, vol. 354, pp. 1771-1775, November 1999.

[3] G.K. Wenning, I. Litvan, and E. Tolosa, "Milestones in atypical and secondary parkinsonisms," Movement Disorders, vol. 26, pp. 1083-1095, May 2011.

[4] L. Brabenec, J. Mekyska, Z. Galaz, and I. Rektorova, "Speech disorders in parkinsons disease: early diagnostics and effects of medication and brain stimulation," Journal of Neural Transmission, March 2017.

[5] K.J. Kluin, N.L. Foster, S. Berent, and S. Gilman, "Perceptual analysis of speech disorders in progressive supranuclear palsy," Neurology, vol. 43, pp. 563-563, 1993.

[6] K.J. Kluin, S. Gilman, M. Lohman, and L. Junck, "Characteristics of the dysarthria of multiple system atrophy," Archives of neurology, vol. 53, pp. 545-548, June 1996.

[7] S. Sachin, G. Shukla, V. Goyal, S. Singh, V. Aggarwal, M. Behari, et al., "Clinical speech impairment in parkinson's disease, progressive supranuclear palsy, and multiple system atrophy," Neurology India, vol. 56, pp. 122, 2008.

[8] S. Skodda, W. Visser, and U. Schlegel, "Acoustical analysis of speech in progressive supranuclear palsy," Journal of Voice, vol. 25, pp. 725-731, November 2011.

[9] Y.E. Huh, J. Park, M.K Suh, S.E. Lee, J. Kim, Y. Jeong, H. Kim, and J.W. Cho, "Differences in early speech patterns between parkinson variant of multiple system atrophy and parkinsons disease," Brain and language, vol. 147, pp. 14-20, May 2015.

[10] J. Rusz, C. Bonnet, J. Klempir, T. Tykalova, E. Baborov, M. Novotny, A. Rulseh, and E. Ruzicka, "Speech disorders reflect differing pathophysiology in parkinsons disease, progressive supranuclear palsy and multiple system atrophy," Journal of neurology, vol. 262, pp. 9921001, 2015.

[11] Gongfeng Li, Khalid Daoudi, Jiri Klempir, and Jan Rusz, "Linear classification in speech-based objective differential diagnosis of parkinsonism," in IEEE-ICASSP - 2018 IEEE International Conference on Acoustics, Speech and Signal Processing, Calgary, Canada, Apr 2018.

[12] N. Miller, U. Nath, E. Noble, and D. Burn, "Utility and accuracy of perceptual voice and speech distinctions in the diagnosis of parkinsons disease, psp and msap," Neurodegenerative Disease Management, vol. 7, pp. 191-203, June 2017.
[13] I. Litvan, Y. Agid, D. Calne, G. Campbell, B. Dubois, R.C. Duvoisin, C.G. Goetz, L. Golbe, J. Grafman, J.H. Growdon, et al., "Clinical research criteria for the diagnosis of progressive supranuclear palsy (steelerichardson-olszewski syndrome) report of the nindsspsp international workshop," Neurology, vol. 47, pp. 1-9, 1996.

[14] S. Gilman, G.K. Wenning, P.A. Low, D.J. Brooks, C.J. Mathias, et al., "Second consensus statement on the diagnosis of multiple system atrophy," Neurology, vol. 71, pp. 670-676, August 2008.

[15] J. Rusz, R. Cmejla, H. Ruzickova, and E. Ruzicka, "Quantitative acoustic measurements for characterization of speech and voice disorders in early untreated parkinsons disease," The journal of the Acoustical Society of America, vol. 129, pp. 350-367, October 2011.

[16] M. Novotny, J. Rusz, R. Cmejla, and E. Ruzicka, "Automatic evaluation of articulatory disorders in parkinson's disease," IEEE/ACM Transactions on Audio, Speech and Language Processing (TASLP), vol. 22, pp. 13661378, September 2014.

[17] J. Rusz, C. Bonnet, J. Klempir, T. Tykalova, E. Baborova, M. Novotny, and E. Ruzicka, "supplementary material: Acoustic measurements for objective evaluation of motor speech disorders.," February 2015.

[18] P. Boersma and D. Weenink, "Praat: doing phonetics by computer [computer program]," Version 5.3.51, 2013.

[19] M. Bruckl, "Vocal tremor measurement based on autocorrelation of contours.," in INTERSPEECH, 2012, pp. 715-718.

[20] G. James, D. Witten, T. Hastie, and R. Tibshirani, An introduction to statistical learning, vol. 112, Springer, 2013. 